\title{
Erratum to: Resonant Compton scattering associated with pair creation
}

\author{
Jeanette I. Weise ${ }^{1}$
}

Published online: 9 September 2015

(C) Springer Science+Business Media Dordrecht 2015

\section{Erratum to: Astrophys. Space Sci. (2014) 351:539-560 DOI 10.1007/s10509-014-1850-x}

I correct typographical errors in the paper Weise (2014). These errors have no effect on the results presented in the paper. I have taken this opportunity to discuss a controversy concerning different frequency factors that appear in the expressions for the cross sections: in Weise (2014) there is a factor $\left(\omega / \omega^{\prime}\right)^{2}$, whereas in Daugherty and Harding (1986), Herold (1979) and Gonthier et al. (2000) the corresponding factor is $\omega / \omega^{\prime}$.

\section{Typographical errors}

There are three equations in Sect. 2.3 which have typographical errors. The first equation is Eq. (24), the left hand side of which should have the subscript $M M^{\prime}$ in the same manner that Eq. (22) does. Consequently, Eq. (24) should read as follows:

$$
\begin{aligned}
\frac{d \sigma_{M M^{\prime}}}{d \Omega}= & \frac{3 \sigma_{T}}{8 \pi} \frac{m^{2} \omega^{2}}{\omega^{\prime 2}} \frac{\omega^{\prime}-\omega+m}{\left(m-\omega \sin ^{2} \theta+\omega^{\prime}\left(1-\cos \theta \cos \theta^{\prime}\right)\right)} \\
& \times \mid e_{M i}^{*} e_{M^{\prime} j}^{\prime} \\
& \times\left\{e^{-i\left(x x^{\prime}\right)^{1 / 2} \sin \psi} \sum_{n^{\prime \prime}}\left[c_{n^{\prime} 0}^{++}\right]_{n^{\prime \prime}}^{i j} e^{-i \psi\left(n^{\prime}-n^{\prime \prime}\right)}\right. \\
& \left.+e^{i\left(x x^{\prime}\right)^{1 / 2} \sin \psi} \sum_{n^{\prime \prime}}\left[d_{n^{\prime} 0}^{++}\right]_{n^{\prime \prime}}^{i j} e^{-i n^{\prime \prime} \psi}\right\}\left.\right|^{2}
\end{aligned}
$$

The online version of the original article can be found under doi:10.1007/s10509-014-1850-x.

\section{J.I. Weise}

weise@physics.usyd.edu.au

1 School of Physics, University of Sydney, NSW 2006, Sydney, Australia
The second is in the second lines of $D_{1}, D_{2}$ and $D_{3}$ in Eq. (28). The $\omega$ symbols in the denominators should be $\omega^{\prime}$ symbols so that Eq. (28) should read as follows:

$$
\begin{aligned}
C_{1} & =\sum_{\epsilon^{\prime \prime}= \pm} \frac{\epsilon^{\prime \prime} A_{1}\left(\epsilon^{\prime \prime},-\right)}{\varepsilon^{\prime \prime}\left(\varepsilon^{\prime}-\epsilon^{\prime \prime} \varepsilon^{\prime \prime}+\omega\right)} \\
& =\frac{2\left(\varepsilon_{0}^{\prime}+m\right)\left\{k_{z}^{\prime}\left(k_{z}^{\prime}-k_{z}\right)+\omega^{\prime}\left(\varepsilon^{\prime}+\varepsilon_{0}^{\prime}\right)\right\}}{\left(\varepsilon^{\prime}+\omega\right)^{2}-\varepsilon^{\prime \prime 2}}, \\
C_{2} & =\sum_{\epsilon^{\prime \prime}= \pm} \frac{\epsilon^{\prime \prime} A_{2}\left(\epsilon^{\prime \prime},-\right)}{\varepsilon^{\prime \prime}\left(\varepsilon^{\prime}-\epsilon^{\prime \prime} \varepsilon^{\prime \prime}+\omega\right)} \\
& =\frac{2\left(\varepsilon_{0}^{\prime}+m\right)\left\{k_{z}^{\prime}\left(k_{z}^{\prime}-k_{z}\right)-\omega^{\prime}\left(\varepsilon^{\prime}+\varepsilon_{0}^{\prime}\right)\right\}}{\left(\varepsilon^{\prime}+\omega\right)^{2}-\varepsilon^{\prime \prime 2}},
\end{aligned}
$$

$C_{3}=\sum_{\epsilon^{\prime \prime}= \pm} \frac{\epsilon^{\prime \prime} B_{3,4}\left(\epsilon^{\prime \prime},-\right)}{\varepsilon^{\prime \prime}\left(\varepsilon^{\prime}-\epsilon^{\prime \prime} \varepsilon^{\prime \prime}+\omega\right)}$

$$
=\frac{2\left(\varepsilon_{0}^{\prime}+m\right)\left(k_{z}^{\prime}-k_{z}\right)}{\left(\varepsilon^{\prime}+\omega\right)^{2}-\varepsilon^{\prime \prime 2}},
$$

$$
D_{1}=\sum_{\epsilon^{\prime \prime}= \pm} \frac{\epsilon^{\prime \prime} A_{1}^{\prime}\left(\epsilon^{\prime \prime},-\right)}{\varepsilon^{\prime \prime}\left(\varepsilon^{\prime}-\epsilon^{\prime \prime} \varepsilon^{\prime \prime}-\omega^{\prime}\right)}
$$

$$
\begin{aligned}
& =\frac{2\left(\varepsilon_{0}^{\prime}+m\right)\left\{k_{z}\left(k_{z}^{\prime}-k_{z}\right)+\omega\left(\varepsilon^{\prime}+\varepsilon_{0}^{\prime}\right)\right\}}{\left(\varepsilon^{\prime}-\omega^{\prime}\right)^{2}-\varepsilon^{\prime \prime 2}}, \\
D_{2} & =\sum_{\epsilon^{\prime \prime}= \pm} \frac{\epsilon^{\prime \prime} A_{2}^{\prime}\left(\epsilon^{\prime \prime},-\right)}{\varepsilon^{\prime \prime}\left(\varepsilon^{\prime}-\epsilon^{\prime \prime} \varepsilon^{\prime \prime}-\omega^{\prime}\right)} \\
& =\frac{2\left(\varepsilon_{0}^{\prime}+m\right)\left\{k_{z}\left(k_{z}^{\prime}-k_{z}\right)-\omega\left(\varepsilon^{\prime}+\varepsilon_{0}^{\prime}\right)\right\}}{\left(\varepsilon^{\prime}-\omega^{\prime}\right)^{2}-\varepsilon^{\prime \prime 2}}, \\
D_{3} & =\sum_{\epsilon^{\prime \prime}= \pm} \frac{\epsilon^{\prime \prime} B_{3,4}^{\prime}\left(\epsilon^{\prime \prime},-\right)}{\varepsilon^{\prime \prime}\left(\varepsilon^{\prime}-\epsilon^{\prime \prime} \varepsilon^{\prime \prime}-\omega^{\prime}\right)} \\
& =\frac{2\left(\varepsilon_{0}^{\prime}+m\right)\left(k_{z}^{\prime}-k_{z}\right)}{\left(\varepsilon^{\prime}-\omega^{\prime}\right)^{2}-\varepsilon^{\prime \prime 2}} .
\end{aligned}
$$


The third is in the second equation of Eq. (29) for the modes $\perp^{\prime} \|$ and $\left\|^{\prime}\right\|$. Note also that Eq. (29) is explicitly written for $\theta^{\prime}=180^{\circ}$. Equation (29) should read:

$$
\begin{aligned}
\frac{d \sigma^{\perp^{\prime} \perp}}{d \cos \theta}= & \frac{d \sigma^{\|^{\prime} \perp}}{d \cos \theta}=\frac{3 \sigma_{T}}{4} \frac{\omega^{2}}{\omega^{\prime 2}} \\
& \times \frac{e^{-x}}{\left(m-\omega \sin ^{2} \theta+\omega^{\prime}+\omega^{\prime} \cos \theta\right)} \\
& \times \frac{\left\{\left(C_{2}\right)^{2}+\left(D_{2}\right)^{2}\right\}}{32\left(\varepsilon^{\prime}+m\right)}, \\
\frac{d \sigma^{\perp^{\prime} \|}}{d \cos \theta}= & \frac{d \sigma^{\left\|^{\prime}\right\|}}{d \cos \theta}=\frac{3 \sigma_{T}}{4} \frac{\omega^{2}}{\omega^{\prime 2}} \\
& \times \frac{e^{-x}}{\left(m-\omega \sin ^{2} \theta+\omega^{\prime}+\omega^{\prime} \cos \theta\right)} \frac{1}{32\left(\varepsilon^{\prime}+m\right)} \\
& \times\left\{\cos ^{2} \theta\left[\left(C_{2}\right)^{2}+\left(D_{2}\right)^{2}\right]\right. \\
& +\omega^{2} \sin ^{4} \theta\left[\left(C_{3}\right)^{2}+\left(D_{3}\right)^{2}\right] \\
& \left.+2 \omega \sin ^{2} \theta \cos \theta\left[\left(C_{2}\right)\left(C_{3}\right)+\left(D_{2}\right)\left(D_{3}\right)\right]\right\} .
\end{aligned}
$$

\section{Frequency factor}

The different frequency factor between my work (Weise 2014) and notably that of Gonthier et al. (2000) arises when the Compton scattering probability is expressed as a differential cross section. A check on which of these factors is correct is provided by considering the Klein-Nishina limit, which involves taking the weak field limit, $B \rightarrow 0$, and considering scattering by an electron initially at rest.

The approach used in Weise (2014), to obtain the differential cross section, follows Stoneham (1980) and Melrose and Sy (1972), viz the conversion formula of Eq. (22). Stoneham, using this conversion formula, evaluated the Compton scattering cross section for $e B / m \ll \omega^{\prime}$ using the proper time technique and correctly obtained the KleinNishina cross section for low frequency photons. While this justifies the factor used in Weise (2014), it would be desirable to demonstrate explicitly that the $S$-matrix approach with this factor reproduces the Klein-Nishina cross section. The main practical difference between the proper-time and $S$-matrix approaches is in the form of the electron propagator. The propagator in the proper-time approach involves an integral over proper time (effectively the gyrophase angle), and in the $S$-matrix approach an expansion in orthogonal polynomials is made and the integral over proper time is carried out explicitly. As a result, the $S$-matrix formula includes an exponential coefficient with argument $-\left(x+x^{\prime}\right)$, with $x=k_{\perp}^{2} / 2 e B$ and $x^{\prime}=k_{\perp}^{\prime 2} / 2 e B$, and $J$-functions (generalized Laguerre functions), with integer orders, related to the
Landau quantum numbers $\left(n, n^{\prime}, n^{\prime \prime}\right)$ of the initial, final and intermediate (virtual) electron states, and with arguments $x$ and $x^{\prime}$. Reproducing the Klein-Nishina limit is then problematic, due to the implicitly assumed azimuthal symmetry about the magnetic field being artificial in the limit $B \rightarrow 0$.

One approach is to set $n=n^{\prime}=n^{\prime \prime}=0$ and then take the limit $B \rightarrow 0$. This corresponds to assuming that the electron is always in its ground (Landau) state. Such an assumption can be justified physically for sufficiently large $B$, but this is incompatible with $B \rightarrow 0$. This approach does not allow one to derive the Klein-Nishina limit.

A second approach requires that the initial electron not be at rest. The initial electron then has a (quantized) perpendicular momentum $p_{\perp} \propto \sqrt{n}$, with $n$ its Landau quantum number. The classical (non-quantum) limit corresponds to $n \rightarrow \infty$ (Melrose and Parle 1983). The sum over $n, n^{\prime}, n^{\prime \prime}$ is replaced by an integral over $p_{\perp}^{\prime \prime}$, say, together with infinite sums over $a=n-n^{\prime \prime}$ and $b=n^{\prime}-n^{\prime \prime}$, and with the $J$ functions replaced with Bessel functions. However, the next step in an attempt to derive the Klein-Nishina limit leads to inconsistency. One needs either to expand the Bessel functions in terms of $z=k_{\perp} p_{\perp}^{\prime \prime} / e B \ll 1$ and $z^{\prime}=k_{\perp}^{\prime} p_{\perp}^{\prime \prime} / e B \ll$ 1 , or expand the original $J$-functions in terms of $x \ll 1$ and $x^{\prime} \ll 1$. Both of these expansions compromise any $B \rightarrow 0$ limit that is necessary to ultimately obtain the low frequency Klein-Nishina cross section. One requires $e B / m \ll \omega^{\prime}$ in taking the limit $B \rightarrow 0$, whereas the approximations to the $S$-matrix formalism require $\omega^{\prime} \ll e B / m \ll m$. No such inconsistency arises when the proper-time approach is used (Stoneham 1980; Milton et al. 1974), as $B \rightarrow 0$ is consistent with $e B / m \ll \omega^{\prime}$ used in the proper-time formalism.

There seems to be no simple way of deriving the KleinNishina limit from the $S$-matrix formalism, in its usual form, by taking $B \rightarrow 0$ and assuming the initial electron to be at rest. The justification for the factor $\left(\omega / \omega^{\prime}\right)^{2}$ in the cross section is that it is required for the derivation of the KleinNishina limit using the proper-time approach.

\section{References}

Weise, J.I.: Astrophys. Space Sci. 351, 539 (2014)

Daugherty, J.K., Harding, A.K.: Astrophys. J. 309, 362 (1986)

Herold, H.: Phys. Rev. D 19, 2868 (1979)

Gonthier, P.L., et al.: Astrophys. J. 540, 907 (2000)

Stoneham, R.J.: Opt. Acta 27, 537 (1980)

Melrose, D.B., Sy, W.: Astrophys. Space Sci. 17, 343 (1972)

Melrose, D.B., Parle, A.J.: Aust. J. Phys. 36, 755 (1983)

Milton, K.A., et al.: Phys. Rev. D 10, 1299 (1974) 